\title{
Konsekutive Aspiration
}

\section{Consecutive Aspiration}

Autor

Institut

\section{A. Hermes}

Onkologischer Schwerpunkt, Krankenhaus Großhansdorf (Leitung: Dr. med. U. Gatzemeier) eingereicht 1.7.2009

akzeptiert nach Revision 6. 7.2009

\section{Bibliografie}

DOI http://dx.doi.org/ 10.1055/s-0029-1214960 Pneumologie 2009; 63: 653-655 @ Georg Thieme Verlag KG Stuttgart · New York ISSN 0934-8387

Korrespondenzadresse Dr. med. Andreas Hermes Onkologischer Schwerpunkt Krankenhaus Großhansdorf Wöhrendamm 80 22927 Großhansdorf a.hermes@kh-grosshansdorf.de

\section{Zusammenfassung $\nabla$}

Wir berichten über einen 40 Jahre alten Patienten, der in den letzten Wochen klinisch rezidivierende Pneumonien mit geringem Ansprechen auf Antibiotika geboten hatte. Computertomografisch wurde der Verdacht auf eine rechts-hiläre Raumforderung mit abhängiger Teilatelektase des Mittellappens gestellt. Mittels starrer Bronchoskopie wurde ein besonderer Fremdkörper aus dem rechten Hauptbronchus geborgen.

\section{Fallbericht \\ $\nabla$}

Der 40 Jahre alte Patient litt seit etwa 15 Jahren an einer chronisch-paranoiden Psychose. Aufgrund seines ausgeprägt fremdaggressiven Verhaltens musste er letztlich in der forensischen Psychiatrie untergebracht werden. Es besteht ein langjähriger Nicotinabusus. Weitere relevante Vorerkrankungen sind nicht bekannt.

In den letzten Wochen war vermehrt produktiver Husten aufgetreten. Verschiedene Antibiotikatherapien erbrachten keine wesentliche Besserung. Ein Aspirationsgeschehen war dem Patienten nicht erinnerlich und wurde auch nicht beobachtet.

Vier Wochen vor der Aufnahme in unserer Abteilung wurde ein thorakales Computertomogramm angefertigt. Es wurde eine rechts-hiläre Raumforderung mit Einengung des zentralen rechten Bronchialsystems sowie einer Teilatelektase des Mittellappens dokumentiert. Zwei Wochen später erfolgte eine ambulante Bronchoskopie mit Darstellung eines den rechten Hauptbronchus okkludierenden Fremdkörpers. Bei der Untersuchung entleerte sich reichlich gelblich-rahmiges Sekret. Der zunehmend agitierte Patient beendigte schließlich die Bronchoskopie selbst.

Bei der stationären Aufnahme präsentierte sich der Patient in einem stabilen, aber leicht reduzierten Allgemeinzustand in Begleitung zweier

\section{Abstract \\ $\nabla$}

We report on a 40 year-old man who presented with recurrent pneumonia with limited effect of antibiotics. A chest CT-scan revealed a tumor in the right hilar region leading to a partial atelectasis of the middle lobe. We removed a rather uncommon foreign body from the right main stem bronchus.

Pfleger der psychiatrischen Abteilung. Die Sprache war weitgehend unartikuliert, intermittierend brüllte der Patient und war sehr agitiert, sodass teilweise eine Fixierung mit Handschellen erforderlich war.

Der Blutdruck betrug 120/ 70 mm Hg, die Pulsfrequenz 88/min, regelmäßig. Die Atemfrequenz lag bei 16/min. Es bestand keine Lippenzyanose. Über den Lungen war seitengleich ein vesikuläres Atemgeräusch zu auskultieren. Der Klopfschall war sonor. Insbesondere über dem basalen Drittel des rechten Hemithorax waren bronchitische Fremdgeräusche hörbar. Der übrige internistische Untersuchungsbefund war unauffällig.

Das Aufnahmelabor zeigte normale Gerinnungswerte, ein leicht erhöhtes Kreatinin $(1,3 \mathrm{mg} / \mathrm{dl})$,

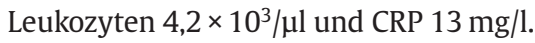

Das Röntgenbild des Thorax p.a. und seitlich rechts anliegend dokumentierte den rechten Hilus basal akzentuiert sowie eine Zeichnungsvermehrung im Bereich des Mittellappens. Der rechte Hauptbronchus erschien proximal okkludiert $($ Abb. 1).

Eine Blutgasanalyse (Kapillarblut aus dem Ohrläppchen) ohne $\mathrm{O}_{2}$-Zufuhr ergab die folgenden Werte: pH 7,44, $\mathrm{pCO}_{2} 44,7 \mathrm{mmHg}, \mathrm{pO}_{2}$ 57,1 $\mathrm{mmHg}$, Base Excess 5,3 mmol/L, $\mathrm{sO}_{2} 91,6 \%$.

Das EKG zeigte eine Sinusbradykardie von $47^{\prime} /$ min bei Steiltyp sowie eine unauffällige Erregungsausbreitung und -rückbildung. 


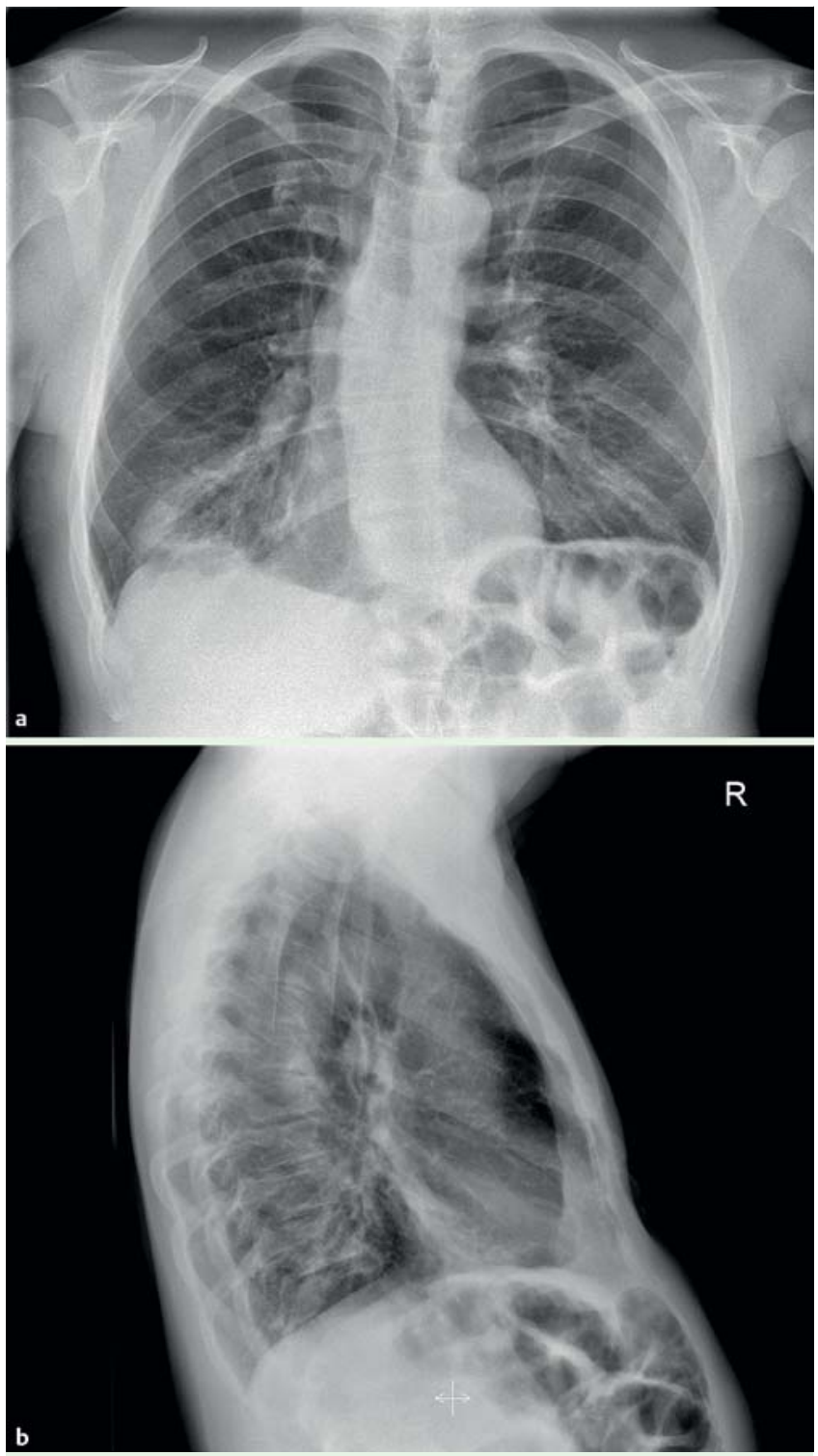

Abb. 1 a Röntgen Thorax p. a. und b seitlich rechts anliegend.

Am Morgen der stationären Aufnahme erfolgte zunächst unter Gabe von Propofol eine orientierende flexible Bronchoskopie mit Darstellung einer von einem blauen Rand umgebenen Erbse im proximalen rechten Hauptbronchus mit subtotaler Stenose. Nebenbefundlich war eine mittelgradige chronische Bronchitis mit geringer Schleimhautrötung zu verzeichnen.

Der Patient wurde sodann starr intubiert und der Fremdkörper mittels einer Krokodilzange erfasst. Nach Entfernung entpuppte sich der Fremdkörper als ein Spielhütchen aus Plastik, in dessen hohler Basis sich zusätzlich eine Erbse befand ( $A$ Abb. 2-4).

Nach der Fremdkörperentfernung entleerte sich erwartungsgemäß eine größere Menge gelbliches Sekret. Bei der anschließenden Inspektion war der rechte Hauptbronchus einschließlich aller Segmentbronchien normal konfiguriert darstellbar.

Eine halbe Stunde nach dem Eingriff befand sich der Patient wieder in seinem üblichen Zustand und wurde in die forensische Abteilung zurückverlegt. Über 10 Tage wurde eine Therapie mit Amoxicillin und Clavulansäure verabreicht.

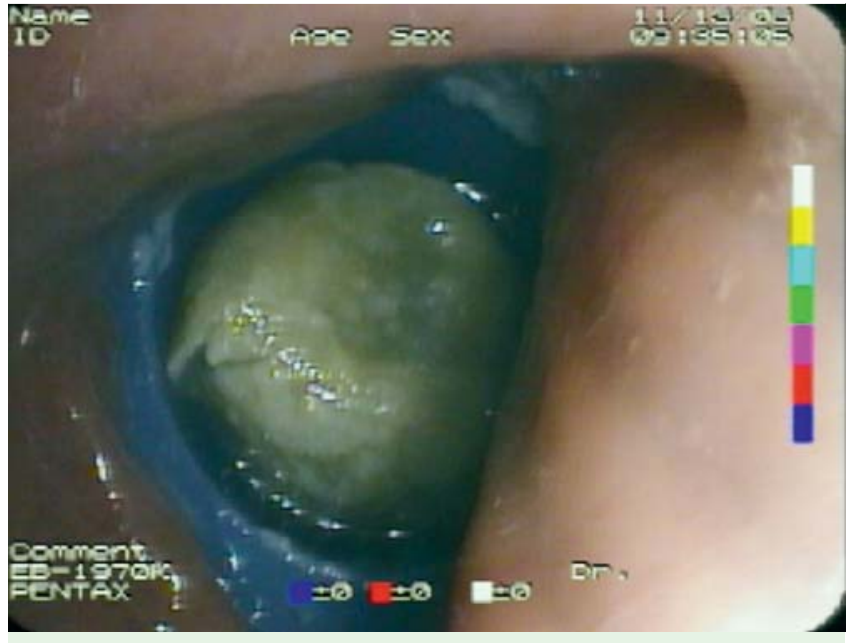

Abb. 2 Fremdkörper im proximalen rechten Hauptbronchus.

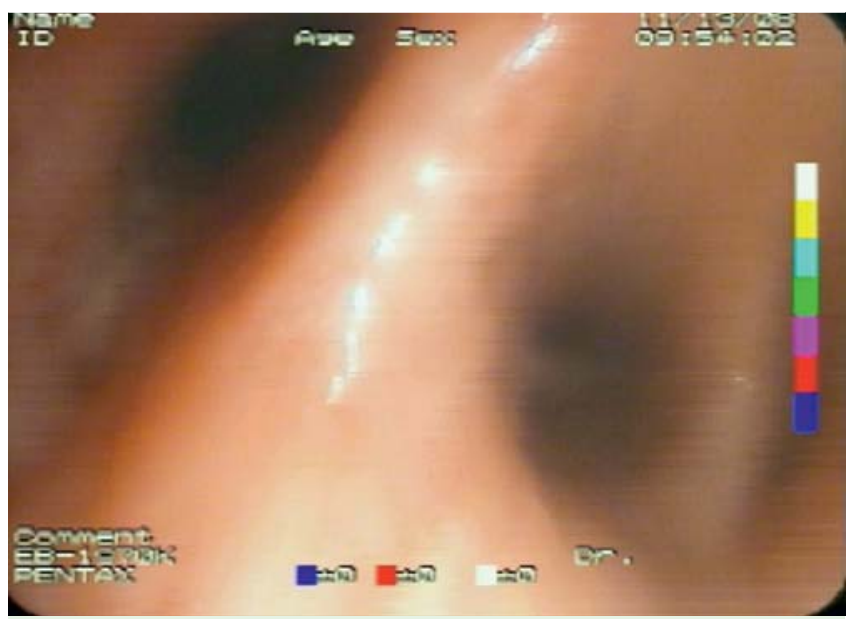

Abb. 3 Distaler Bronchus Intermedius nach Fremdkörperextraktion.

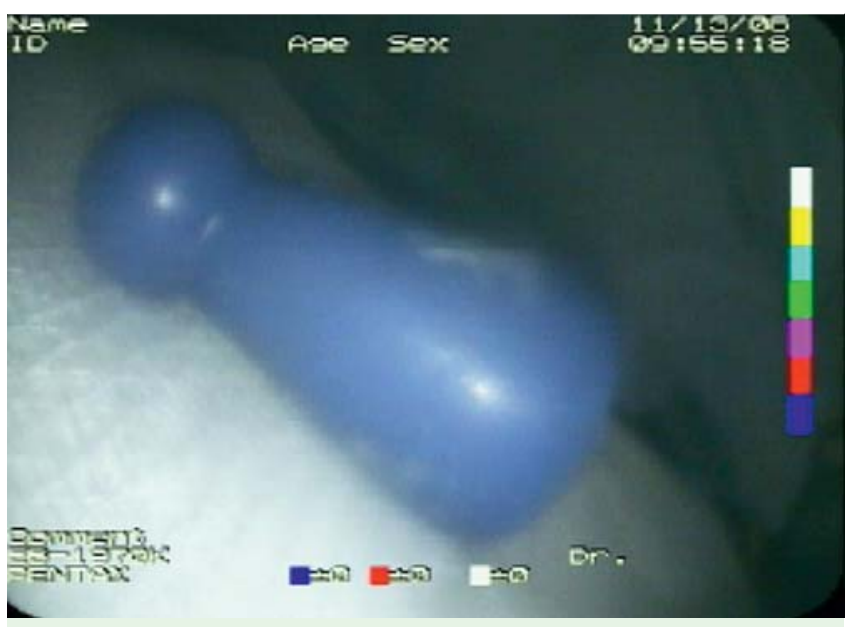

Abb. 4 Fremdkörper. 
Die Fremdkörperaspiration ist insbesondere im Kindesalter eine häufigere pneumologische Diagnose. Im Erwachsenenalter kommt es insbesondere bei Vigilanzstörungen (z. B. bei Alkoholintoxikation, Drogen- oder Medikamenteneinnahme im Rahmen eines Krampfanfalles, Synkopen, Schädel-Hirn-Trauma) sowie bei Erbrechen insbesondere in Rückenlage zu Aspirationen. Akzidenzielle oder auch bewusste Fremdkörperaspirationen treten insbesondere bei Patienten mit psychiatrischen Grunderkrankungen gehäufter auf.

Aufgrund der anatomischen Verhältnisse erfolgt eine Fremdkörperaspiration häufiger in das rechte Bronchialsystem.

Die wichtigsten Komplikationen sind die Asphyxie durch Verlegung der zentralen Atemwege, (rezidivierende) Pneumonien und/oder Atelektasen.

Die Entfernung des Fremdkörpers erfolgt üblicherweise über eine starre Bronchoskopie, wie auch bei dem beschriebenen Patienten. Lediglich bei kleineren, nicht scharfen oder spitzen Fremdkörpern kann über eine flexible Bronchoskopie mit entsprechendem Instrumentarium eine Extraktion versucht werden [1-4].

Die Besonderheit des beschriebenen Falles liegt in der offensichtlich konsekutiven Aspiration zweier Fremdkörper in den rechten proximalen Hauptbronchus.

\section{Interessenkonflikte}

$\nabla$

Es besteht kein Interessenkonflikt.

\section{Literatur}

1 Dobbertin I, Dierkesmann R, Kohlhäufl M. Entfernung von Fremdkörpern. Lehrbuch und Atlas der Bronchoskopie, 2. Auflage. Bern: Huber, 2008: $347-348,359-363$

2 Lan RS, Lee CH, Chiang YC, Wang WJ. Use of fiberoptic bronchoscopy to retrieve bronchial foreign bodies in adults. Am Rev Resp Dis 1989; 140 : $1734-1737$

3 Limper AH, Prakash UBS. Transbronchial foreign bodies in adults. Ann Intern Med 1990; 112: 604

4 Mehta AC, Dasgupta A. Bronchoscopic approach to tracheobronchial foreign bodies in adults. Pro-flexible Bronchoskopy; Journal of Bronchology 1997; 4: 173-178

\section{Buchbesprechung}

\section{Checkliste Tauchtauglichkeit}

Tetzlaff K, Klingmann C, Muth C et al. (Hrsg)

1. Auflage 2008. Stuttgart: Gentner Verlag, 384 S. geb., $30,00 €$

ISBN 978-3-87247-681-4

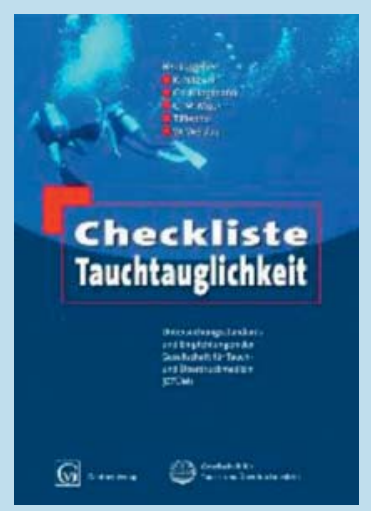

Der Tauchsport erfreut sich in Deutschland nach wie vor größter Beliebtheit. Das zunehmende Alter der aktiven Sportler geht dabei Hand in Hand mit einer erhöhten Prävalenz von Krankheiten. Diese in Zusammenhang mit den besonderen physikalischen Belastungen zu stellen, denen der Betreffende bei der Ausübung seines Sportes in einer prinzipiell lebensfeindlichen Umgebung ausgesetzt ist, macht die außerordentliche Herausforderung aus, der sich der Arzt stellt, der eine Tauchtauglichkeitsuntersuchung durchführt.

Mit der Checkliste Tauchtauglichkeit wurde nun endlich ein umfassendes Handbuch vorgelegt, das zu allen Fragen, die in diesem Zusammenhang auftreten, ausführlich Stellung nimmt und zudem, da unter der Schirmherrschaft der GTÜM und ÖGTH erschienen, den Charakter einer Leitlinie besitzt. Eingegangen wird auf jedes potenzielle medizinische Problem, das im Zusammenhang mit dem Tauchsport auftreten kann. Von der Augen- bis zur Zahnheilkunde wird in Spezialkapiteln intensiv diskutiert, welchen Fragestellungen sich der Arzt dabei im Einzelnen gegenüber sehen kann:

„Wann besteht bei einem Patienten mit kutanem T-ZellLymphom Tauchtauglichkeit?"

„Welcher Patient mit Herzrhythmusstörungen darf tauchen, welcher nicht?“

„Nach wie vielen anfallsfreien Jahren darf einem Epileptiker eine eingeschränkte Taucherlaubnis erteilt werden?“

In gesonderten Kapiteln wird außerdem auf besondere Problemfelder eingegangen, wie etwa „Tauchen bei bestimmten Altersgruppen“, „Tauchtauglichkeit bei Behinderten“ oder bei „Patienten mit Tumorerkrankungen“.

Didaktisch folgt die Checkliste Tauchtauglichkeit eher einem traditionellen Konzept. Abbildungen, Flussschemata oder Fallbeschreibungen sucht man vergebens. Auch wirkt der Text teilweise recht trocken, ohne je durch Anekdoten oder historische Verweise aufgelockert zu werden. Denkbar wäre etwa ein Hinweis auf die römischen Kampftaucher („Urinatores“ genannt) oder auf Leni Riefenstahl, die in hohem Alter noch ihren ersten Tauchgang wagte. Aufgewogen wird dies durch das klare und übersichtliche didaktische Konzept.

Zusammenfassend liegt hier nun endlich ein Referenzwerk vor, das dem tauchmedizinisch tätigen Arzt klare Konzepte und Antworten auf alle seine Fragen liefert.

Ingo Finnmann, Rotenburg 